\title{
MULTIPLE CAVITY EXPERIMENTS TO DETECT PARITY NONCONSERVATION IN ATOMIC HYDROGEN
}

\author{
R.T. ROBISCOE * \\ Lab. für Kernphysik, ETH-Zürich, CH8093 Zürich, Switzerland
}

and

\author{
W.L. WILLIAMS \\ Physics Department, University of Michigan, Ann. Arbor, Michigan 48109. U.S.A.
}

Received 15 September 1981

We develop general guidelines and criteria for designing and evaluating beam experiments which use Ramsey's method of separated oscillating fields to detect PNC (parity nonconserving) effects in atomic hydrogen. We find that variation of the relative radio-frequency phases between different field configurations may offer distinct advantages in measuring and processing expected PNC data. We evaluate several specific experiments employing such multiple region designs.

\section{Introduction}

The "electroweak" theory of Weinberg and Salam has predicted and successfully accounted for a variety of PNC (parity nonconserving) effects which have been observed in high energy scattering experiments [1]. The theory also predicts PNC processes in the low energy realm of atomic physics [2]; here, however, experimental verification is not yet conclusive although experimental results with bismuth and thallium favor some PNC effect [3]. The search continues for PNC effects in atoms [4]. The ongoing atomic experiments have at least two objectives: (1) to verify the underlying unity of the theory by observing PNC interactions in the low energy regime, thus complementing the high energy results, (2) to bring to bear on the problem the high-precision methods of atomic physics, in the hope of measuring the characteristic coupling constants of the theory either more accurately or in more detail than has been done in the high energy experiments.

Regarding the latter objective, PNC experiments in atomic hydrogen and deuterium appear to have several advantages in principle over experiments on heavy atoms. First, the PNC effects are exactly calculable for the one-electron hydrogen atom. By comparison, calculation for heavy atoms are somewhat ambiguous because of the inherent difficulties of the many electron problem. Second, experiments on hydrogen and de-

\footnotetext{
* Permanent address: Physics Department, Montana State
} University, Bozeman, Montana 59717, U.S.A. uterium can measure - with about equal sensitivity - all four coupling constants which occur in Weinberg-Salam theory (for atoms, these are the four constants specifying the neutral weak current couplings between electron and proton, and electron and neutron, both nucleon spin-independent and spin-dependent). The heavy atom experiments are sensitive primarily to the two spinindependent electron-nucleon couplings. It is fair to note that this does include what is very probably the dominant PNC interaction in atoms, namely the spinindependent electron-neutron coupling. The other three coupling constants appear to be smaller than this one by about an order of magnitude. Nevertheless, if information is to be gained on the spin-dependent couplings, it may come most easily from the atomic hydrogen PNC experiments.

Other advantages for the hydrogen experiments may result from the experimental method used: for the experiments under way, this is the atomic beam resonance method. Here an intense, well-collimated, monoenergetic beam of $2 \mathrm{~S}$ metastable hydrogen atoms in a given hfs (hyperfine structure) state traverses a succession of well-defined electromagnetic fields (both static and oscillatory electric and magnetic fields may be used) which induce transitions to another $2 \mathrm{~S}$ hfs state that can be selectively detected downstream. Systematic effects can be controlled and measured relatively easily; they can be simulated where necessary by application of additional fields. It is also readily possible to construct field geometries exhibiting a variety of pseudoscalar quantities; these occur in combination with the PNC 
terms of interest, so that the latter may be isolated by several signal substractions following reversals of the appropriate fields. Some of these features also exist in experiments on heavy atoms. However, in these experiments, the sample atoms usually are contained in a hot vapor cell, where environmental systematic effects may be more difficult to deal with, and where successive application of different, phase-coherent fields in a fixed time sequence would be technically difficult and would appear to lead to few easily measurable effects.

For all these reasons, it is worth pursuing the hydrogen atomic beam PNC experiments, and improving and diversifying them where possible. In the latter regard, the notion of using relative field phases to add to the choice of experimental pseudoscalar "subtraction variables" necessary in all PNC experiments is a natural one for beam experiments, because the sample atoms can travel through a succession of interaction regions, where the fields have preset and precisely controlled relative phases. Since the fields operate continuously, and are turned "on" and "off" in the rest frame of the atom by its unidirectional motion through the field regions, problems with a time sequence are automatically circumvented. The general idea of using separated field regions with adjustable relative phases as a spectroscopic tool in beam experiments was suggested some time ago by Ramsey [5] and the technique has been used successfully in a number of applications. It is only in the last few years, however, that the use of this technique has been discussed in specific instances in connection with the hydrogen PNC experiments [6]. In certain cases, there are some advantages in principle to be gained by use of the Ramsey technique, but there appear to be no general guidelines as to how the technique might be employed most effectively in the hydrogen PNC work.

In this paper, we hope to supply some of those guidelines by discussing the general features and limitations of the Ramsey technique as applied to hydrogen PNC experiments, and its advantages and disadvantages relative to experiments employing single field regions. In what follows, we shall use the word "cavity" in place of "field (or interaction) region", since the fields of interest are most often generated inside resonant cavities. Our goal is to assess what advantages may be gained or new information found in going from single cavity to multiple cavity experimental schemes.

In section 2, we discuss some standards of comparison between various hydrogen PNC experiments, in order to establish criteria which should be met by any new experimental scheme. Some advantages and disadvantages of multiple cavity experiments are immediately apparent. Next, in section 3, we derive an approximate quantum mechanical solution for a multiple cavity atomic beam experiment, and we use it to point out general features of such experiments. Although couched in terms of hydrogen PNC experiments, our solution is of some general utility. In section 4, we analyze several specific multiple cavity experiments which promise some improvements over single cavity schemes. Our conclusions regarding the general utility of such experiments appear in sect. 5 .

\section{Standards of comparison}

Before dealing with multiple cavity hydrogen PNC experiments, it is worth discussing some of the standards achieved in a single cavity experimental design and operation, since any new scheme should match or exceed these standards. The standards we are interested in here relate to: (1) availability and control of the experimental pseudoscalars used to isolate the PNC term, (2) main signal counting rate, (3) experimental sensitivity - i.e., the ratio of the desired PNC signal to the main signal, and (4) integration time - i.e., the data acquisition time necessary to measure the PNC term at a signal-to-noise ratio of unity. Such standards can be judged on a numerical basis for hydrogen PNC experiments in general. We have not included systematic errors here since they usually must be judged on a case-by-case basis.

To make the discussion quantitative, we note that at the heart of any parity experiment is the measurement of the rate of some process in an apparatus which breaks inversion symmetry, and that the amplitude for such a process is separable into a parity conserving (PC) part and a parity nonconserving (PNC) part. We represent this by the total amplitude: $\beta=A_{\mathrm{PC}}+A_{\mathrm{PNC}}$, where the signs imply that under inversion-accomplished experimentally by reversing some pseudoscalar variable the $\mathrm{PC}$ and PNC amplitudes have different relative signs. Since $A_{\mathrm{PNC}}$ is expected to be very small relative to $A_{\mathrm{PC}}$, the intensity or rate for the process can be written as

$|\beta|^{2}=R \pm J$

where $R=\left|A_{\mathrm{PC}}\right|^{2}$ is the main signal strength, and $J=$ $2 \operatorname{Re}\left(A_{\mathrm{PC}}^{*} A_{\mathrm{PNC}}\right)$ is a small interference term which contains the desired PNC information. The PNC term $J$ can be isolated by forming the signal difference upon reversal of the experimental pseudoscalar, which we call a "subtraction variable", as: $\Delta|\beta|^{2} \simeq 2 J+\Delta R$. Here $\Delta R$ is a residual from the main signal which may result from an imperfect reversal of the subtraction variable. In these terms, we can now discuss the standards of comparison noted above for various hydrogen $\mathrm{PNC}$ experiments.

With regard to subtraction variables, we begin by noting that the PNC term $J$ is fractionally very small compared to the main signal $R$ : even in a favorable case [7], we have only: $J / R \sim 10^{-6}$. This has several consequences. First, since there is little hope of reliably 
measuring $1 \mathrm{ppm}$ effects on $R$ directly, we must in fact look for $J$ by exploiting its pseudoscalar signature. That is, we need the subtraction variables a priori to provide the sign reversals which isolate $J$ and $R$. Second, reversal of the subtraction variables must be controlled accurately to avoid generating residuals $\Delta R$ which overwhelm $J$. If a given reversal generates a $\Delta R$ which is only $0.1 \%$ of the main signal, this $\Delta R$ is still $\sim 10^{3}$ times larger than $J$. Such an unfavorable ratio can only be reduced by further reversals; evidently it is desirable to have available more than one subtraction variable. Finally, to avoid residuals associated with long term signal drifts, the subtraction variables should be capable of being rapidly cycled; for beam experiments, electronic cycling at frequencies $\geqslant 1 \mathrm{~Hz}$ is desirable.

In various proposed single cavity hydrogen PNC experiments [7-10], several subtraction variables (usually three) are used. One is a Zeeman field $B_{Z} \sim 575 \mathrm{G}$ in which transitions are driven between $2 \mathrm{~S}$ hfs levels near the crossing point between the states $\beta\left(2 \mathrm{~S}, m_{J}=\right.$ $-1 / 2)$ and $\mathrm{e}\left(2 \mathbf{P}, M_{J}=+1 / 2\right)$, where the PNC coupling between $\beta$ and $\mathrm{e}$ is enhanced. Rapid cycling of $B_{\mathrm{Z}}$ is difficult, but frequencies $-1 \mathrm{~Hz}$ can be achieved. To avoid unsuitably large main signal residuals, the $B_{Z}$ reversal must be controlled to within $\sim 50 \mathrm{ppm}$ (in both magnitude and direction), which should be attainable. In these terms, the Zeeman field is an acceptable subtraction variable. The other subtraction variables may be various static electric field components, or the component of an if (radio-frequency) field along a given axis. The static fields can be rapidly cycled, but complicated asymmetries may appear in the main resonance signal $R$ if the field intensities change along the beam path [11]. Reversing the component of an rf field along a given axis may require a mechanical rotation of the cavity, which cannot be rapidly cycled, or it may require operating the cavity in more than one resonant mode, where field distributions may change, and strict control of relative power levels is required. Thus, in the single cavity hydrogen PNC experiments, the subtraction variables used in addition to the Zeeman field may have some shortcomings with regard to either rapid cycling or to control of possible asymmetrizing effects on the main resonance signal $R$.

At this point, a possible technical advantage for multiple cavity experiments becomes apparent. If we can use relative rf field phases as subtraction variables, then rapid and accurate cycling is possible [12], and since phase reversals (or phase shifts) in an rf field are not expected to change its intensity distribution within an rf cavity, the cycling should have few, if any, asymmetrizing effects on the signal $R$. So long as the cavities are independent of one another, control of power levels during phase reversals is not even particularly critical. In these respects, if phase subtraction variables may prove to be superior to those already described.
With regard to the second standard mentioned above, namely the counting rate for the main signal $R$, we note that the single cavity experiments generally have an $R$ which is only a small fraction of the available total $2 \mathrm{~S}$ intensity: $R<10 \mathrm{ppm}$ is typical [7]. This is because the $2 S$ transitions studied are only weakly allowed, and because they are usually driven by and in the presence of electric fields which cannot be made large without quenching the $2 S$ atoms. In respect of this multiple cavity experiments may again provide an advantage. To increase $R$, we can generate the PC amplitude for a given $2 S \rightarrow 2 S^{\prime}$ transition in a cavity designed to reduce electric field quenching of the metastables, so that this cavity can be run at higher power levels to produce a larger transition rate. The $2 \mathrm{~S} \rightarrow 2 \mathrm{~S}^{\prime}$ PNC amplitude, which requires the presence of electric fields and is necessary for the interference term $J$, can then be generated in a second cavity where the $2 S^{\prime}$ quenching is reduced. In this way, the resultant $2 S^{\prime}$ intensity is increased, and calculated $R$ values $>10 \mathrm{ppm}$ are easily obtained. This can be achieved without appreciably changing the experimental sensitivity, $J / R \sim 10^{-6}$.

The third standard of interest is the experimental sensitivity, i.e. the size of the PNC term relative to the main signal. This is

$S=J / R=2 \operatorname{Re}\left(A_{\mathrm{PNC}} / A_{\mathrm{PC}}\right)$.

$S$ should be made as large as possible, so that the PNC term $J$ will not be overwhelmed by possible systematic errors in the main signal $R$. Unfortunately, we must begin with a number which is very small. The intrinsic sensitivity of a PNC experiment in the $n=2$ level of atomic hydrogen is measured by the fractional admixture of opposite parity $2 \mathrm{~S}$ and $2 \mathrm{P}$ states via the neutral weak current interaction. This gives $S \sim 10^{-11}$ for mixing of the $\beta_{0}\left(2 \mathrm{~S}, F=0, m_{F}=0\right)$ and $\mathrm{e}_{0}(2 \mathrm{P}, F=1$, $\left.m_{F}=0\right)$ states, where we have assumed a coupling constant of order unity (i.e., of order $C_{\mathrm{ln}}$, the spinindependent electron-neutron coupling), and have normalized to the $2 \mathrm{~S}_{1 / 2}-2 \mathrm{P}_{1 / 2}$ energy difference (Lamb shift) at zero magnetic field. Fortunately, this forbiddingly small number can be increased substantially by several experimental ploys, which are being used in the extant single cavity hydrogen PNC experiment.

First, Lewis and Williams have pointed out that by working near the $\beta_{0}-\mathrm{e}_{0}$ crossing point on the Zeeman diagram $\left(B_{Z} \simeq 552 \mathrm{G}\right)$, the parity mixing between these states can be enhanced [8]. Second, by selecting appropriate transitions and field geometries, we can suppress $A_{\mathrm{PC}}$ in eq. (2) relative to $A_{\mathrm{PNC}}$. This point has been emphasized by Dunford et al. [7]. Third, we can adjust the fields which generate $A_{\mathrm{PNC}}$ so as to optimize it. Hinds has shown [13] that then $A_{\mathrm{pNC}} \propto \sqrt{\tau}$, where $\tau$ is the beam transit time through the cavity, so that the sensitivity may be increased by lengthening the cavity. He has also shown that this optimization gives the same 
enhancement in $A_{\mathrm{PNC}}$ as working at a crossing point, even at different Zeeman fields. In any case, practical limits to these experimental techniques produce sensitivities which are at best $S \sim$ few ppm, as we have noted and used above. Although this is still a small number, it represents an increase by five orders of magnitude over the intrinsic sensitivity, so experimental ingenuity is not lacking.

Further significant increases in the sensitivity $S$ in going from single cavity to multiple cavity hydrogen PNC experiments do not appear to be readily possible. This is because the enhancement techniques described above are essentially equally applicable to single and multiple cavity configurations, and because introduction of relative field phases as subtraction variables - which is the major new feature of multiple cavity schemes - is mainly a convenient way of changing relative field orientations. Such field reorientations are already possible in the single cavity designs. Some small advantage for multiple cavity designs may result from being able to produce the amplitudes $A_{\mathrm{PC}}$ and $A_{\mathrm{PNC}}$ of eq. (2) in separate cavities, so that each may be varied independently, and the ratio $A_{\mathrm{PNC}} / A_{\mathrm{PC}} \propto S$ optimized with more flexibility. Altogether, however, we anticipate no major breakthrough in sensitivity for multiple cavity schemes. An $S$-value of a few ppm appears to be the current limit of ingenuity for hydrogen PNC experiments.

The final standard of comparison for these experiments is the integration time per data point, i.e., the counting time necessary to measure the PNC term $J$ relative to the main $\operatorname{signal} R$ at signal-to-noise ratio unity. If the total detectable metastable beam flux is $f$ (atoms $/ \mathrm{s}$ ), this time is

$T=R / f J^{2}=1 / f R S^{2}=1 / 4 f\left|A_{\mathrm{PNC}}\right|^{2} \cos ^{2} \Phi$,

in the shot noise limit. Here $\Phi$ is the relative phase between the PC and PNC amplitudes. Evidently we wish to minimize $T$, particularly since the integration time in an actual experiment will exceed this estimate because of unaccounted sources of systematic noise. An intense metastable beam source is required: this is provided by a duoplasmatron ion source followed by a cesium charge exchange cell, which yields metastable fluxes up to $f \sim 10^{13} / \mathrm{cm}^{2} \cdot \mathrm{s}$. Then, for single cavity experiments, with optimum fractional signals $R \sim 5 \mathrm{ppm}$ and sensitivities $S \sim 2$ to $3 \mathrm{ppm}$, we have an integration time $T \sim 1 \mathrm{~h} / C^{2}$, where $C$ is the operative neutral weak current coupling constant. The nominal value $T \sim 1 \mathrm{~h}$ for $C \sim 1$ will be increased not only by additional noise sources, but also by any decrease in $C$. As we have noted in sec. 1 , in the current state of Weinberg-Salam theory, we expect $C \ll 1$ for all but the spin-independent electron-neutron coupling.

As in the case of experimental sensitivity, we do not expect any dramatic change in the integration time $T$ when we go from single to multiple cavity experiments. The reasons are much the same - the available experimental techniques for minimizing $T$ are about equally applicable to both schemes. A marginal advantage for multiple cavity experiments may be that the relative phase $\Phi$ in $T$ of eq. (3) can be more easily optimized, since - with appropriate design - the PC and PNC amplitudes can be produced in separate cavities and independently varied.

To summarize this section, we can say that the following standards should be met by any acceptable hydrogen beam PNC experiment: (1) several experimental pseudoscalar "subtraction variables" should be available to isolate the PNC term $J$ from the main signal $R$, and they should be capable of being accurately and rapidly cycled, (2) the main signal $R$ should be suppressed relative to $J$, but (as a fraction of the total available hydrogen beam) should be $R \sim 10 \mathrm{ppm}$ or larger, (3) the experimental sensitivity $S=J / R$ should be $S \sim$ few ppm or larger (for an assumed PNC coupling constant $C \sim 1$ ), and (4) the integration time $T=$ $R / f J^{2}$ should be $T \sim 1 \mathrm{~h} / C^{2}$ or smaller. We have argued qualitatively that in going from single to multiple cavity experiments, with the introduction of relative of field phases as subtraction variables, we may gain advantages in categories (1) and (2), and perhaps slight improvements in categories (3) and (4). In the next section, we provide a more quantitative basis for these arguments by solving the quantum mechanical problem for a multiple cavity beam experiment.

In passing, we note that because the various fields needed to generate $A_{\mathrm{PC}}$ and $A_{\mathrm{PNC}}$ are separable for multiple cavities, the individual cavity design can be simpler than for a single cavity experiment. It is not necessary to put more than one field into a given cavity - e.g., we do not necessarily need dc field electrodes in an rf cavity. This advantage is tempered somewhat by the fact that in a multiple cavity experiment we must line up the cavities very carefully and must make certain that they operate independently (i.e., are not coupled).

\section{Solution to the multiple cavity problem}

In this section, we derive a quantum mechanical solution suitable for an approximate analysis of multiple cavity hydrogen beam PNC experiments. Our solution is a first order approximation for the final state amplitude in a two-level problem where a beam of atoms, initially in a given state $\alpha$, traverses a succession of cavities in which localized fields induce transitions to a final state $\beta$. In the hydrogen PNC experiments, the states $\alpha$ and $\beta$ are $2 \mathrm{~S}$ hfs levels; we allow them to have arbitrary (induced) decay rates $\gamma_{\alpha}$ and $\gamma_{\beta}$. Also, we allow the cavity fields to have arbitrary time dependence ( $\mathrm{rf}$ and/or static) and spatial dependence along the beam 
path. A first order approximation is justified because, in the relevant experiments, the $\alpha \rightarrow \beta$ transition rate is suppressed to provide sufficient sensitivity to the expected PNC effects. The two-level approximation is adequate when the states $\alpha$ and $\beta$ are relatively weakly coupled to other states (principally $2 \mathrm{P}$ states) and if we include the phenomenological decay rates $\gamma_{\alpha}$ and $\gamma_{\beta}$ as well as allowing for state admistures due to the intrinsic PNC coupling. Although approximate, our solution suffices to show the general features of multiple cavity experiments, especially the role played by field phases.

\subsection{The final state amplitude}

In fig. 1 , we show a diagram for the single cavity problem. States $\alpha$ and $\beta$, with Bohr energies $\hbar \omega_{\alpha}$ and $\hbar \omega_{\beta}$ and decay rates $\gamma_{\alpha}$ and $\gamma_{\beta}$, are coupled by a time-dependent interaction $V(t)$. In Schrödinger representation, the amplitude equations are

$\mathrm{i} \dot{A}_{\alpha}=\epsilon_{\alpha} A_{\alpha}+V_{\alpha \beta}(t) A_{\beta}, \quad \mathrm{i} \dot{A}_{\beta}=\epsilon_{\beta} A_{\beta}+V_{\beta \alpha}(t) A_{\alpha}$.

Here $V_{\alpha \beta}(t)=\langle\alpha|V(t)| \beta\rangle$ is the time-dependent matrix element between the states, and the effect of decay is accounted for in the manner of Bethe-Lamb theory [14] by defining complex energies $\epsilon_{k}=\omega_{k}+\frac{1}{2} i \gamma_{k}$. For a solution to lowest order, we assume that (except for decay) the initial $\alpha$ state amplitude $A_{\alpha}$ remains of order unity, and we solve for the final $\beta$ state amplitude $A_{\beta}$ to order $V$. Then, if the beam enters the cavity at time $t_{0}$ and spends time $\tau$ inside, the emergent amplitudes become

$$
\begin{aligned}
& A_{\alpha}\left(t_{0}+\tau\right) \simeq A_{\alpha}\left(t_{0}\right) \mathrm{e}^{-\mathrm{i} \epsilon_{\alpha} \tau}, \\
& A_{\beta}\left(t_{0}+\tau\right) \simeq A_{\beta}\left(t_{0}\right) \mathrm{e}^{-\mathrm{i} \epsilon_{\beta} \tau}+C_{\beta \alpha}(\tau) A_{\alpha}\left(t_{0}\right) \mathrm{e}^{-\mathrm{i} \epsilon_{\beta} \tau},
\end{aligned}
$$

where

$$
\begin{aligned}
& C_{\beta \alpha}(\tau)=-\mathrm{i} \int_{0}^{\tau} V_{\beta \alpha}\left(t_{0}+t\right) \mathrm{e}^{\mathrm{i} \epsilon_{\alpha \beta} t} \mathrm{~d} t, \\
& \epsilon_{\alpha \beta}=\epsilon_{\alpha}-\epsilon_{\beta}=\omega_{\alpha \beta}-\frac{1}{2} \mathrm{i} \gamma_{\alpha \beta}, \gamma_{\alpha \beta}=\gamma_{\alpha}-\gamma_{\beta} .
\end{aligned}
$$

Here we have assumed that the complex energies $\epsilon$ are

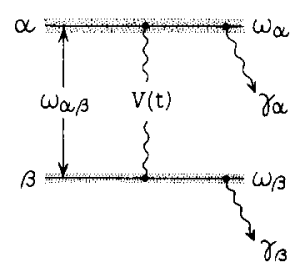

Fig. 1. The two level problem in a single cavity. States $\alpha$ and $\beta$ are separated in energy by $\hbar \omega_{\alpha \beta}=\hbar\left(\omega_{\alpha}-\omega_{\beta}\right)$ and have phenomenological decay rates $\gamma_{\alpha}$ and $\gamma_{\beta}$. They are coupled by a time-dependent interaction $V(t)$. constant over the extent of the cavity. If they are not, then the quantum mechanical phase $\epsilon \tau$ must be replaced by $\int_{0}^{\tau} \epsilon(t) \mathrm{d} t$.

In the approximation of eq. (5), the final $\beta$ state amplitude consists of two parts: the first represents $\beta$ 's freely passing through the cavity (except for decay), and the second results from transitions $\alpha \rightarrow \beta$ induced by the cavity coupling factor $C_{\beta \alpha}(\tau)$. This pattern is repeated after $N$ cavities. If we denote the final state amplitude after $N$ cavities by $\beta_{N}=A_{\beta}\left(t_{0}+\tau_{1}+\ldots+\tau_{N}\right)$, with $\tau_{n}$ the transit time for the $n$th cavity, and let the coupling factor for the $n$th cavity be $C_{n}$, then, for initial conditions $A_{\alpha}\left(t_{0}\right)=1, A_{\beta}\left(t_{0}\right)=0$,

$\beta_{N} \simeq\left\{\sum_{n=1}^{N} C_{n} \exp \left[-\mathrm{i} \sum_{k=1}^{n-1} \delta_{k}\right]\right\} \exp \left[-\sum_{n=1}^{N} \zeta_{n}\right]$

Here $\delta_{k}$ is the quantum mechanical phase difference accumulated between the $\alpha$ and $\beta$ during traversal of the $k$ th cavity

$\delta_{k}=\left(\omega_{\alpha \beta}-\frac{1}{2} \mathrm{i} \gamma_{\alpha \beta}\right)_{k \mathrm{C}} \tau_{k}+\left(\omega_{\alpha \beta}-\frac{1}{2} \mathrm{i} \gamma_{\alpha \beta}\right)_{k \mathrm{D}} T_{k}$,

with $T_{k}$ the transit time for a field free drift region (D) following the $k$ th cavity (C). The geometry for such an experiment appears in fig. 2 . Both the cavity coupling factors $C_{n}$ and complex state energies may change from cavity to cavity. Because we have included decay, the phases $\delta_{k}$ are complex in general. The last parameter in eq. (7) is written as

$\zeta_{n}=\left(\frac{1}{2} \gamma_{\beta}+\mathrm{i} \omega_{\beta}\right)_{n \mathrm{C}} \tau_{n}+\left(\frac{1}{2} \gamma_{\beta}+\mathrm{i} \omega_{\beta}\right)_{n \mathrm{D}} \mathrm{T}_{n}$,

emphasizing that it measures the decay of the $\beta$ amplitude in the $n$th cavity.

The factor containing $\zeta_{n}$ in eq. (7) gives an overall attenuation factor $a_{N}$ for the decay of the $\beta$ state through $N$ cavities. We write

$$
\left|\beta_{N}\right|^{2} \simeq a_{N}\left|\sum_{n=1}^{N} C_{n} \exp \left[-\mathrm{i} \sum_{k=1}^{n-1} \delta_{k}\right]\right|^{2},
$$

where

$a_{N}=2 \operatorname{Re} \sum_{n=1}^{N} \zeta_{n}=\sum_{n=1}^{N}\left[\left(\gamma_{\beta} \tau\right)_{n \mathrm{C}}+\left(\gamma_{\beta} T\right)_{n \mathrm{D}}\right]$

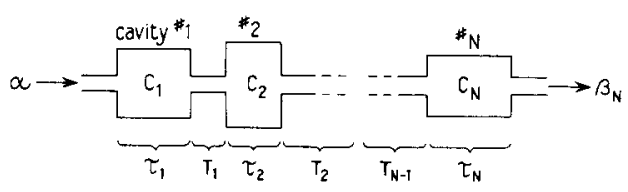

Fig. 2. The $N$ cavity experiment. A beam of atoms, initially in the $\alpha$ state, enters a succession of cavities where various independent fields with couplings $C_{1}, C_{2}, \ldots, C_{N}$ induce $\alpha \rightarrow \beta$ transitions. The $n$th cavity has a beam transit time $\tau_{n}$ and is followed by a field free drift region of transit time $T_{n}$. The emergent $\beta$ state amplitude is denoted by $\beta_{N}$ 
Neither the attenuation factor $a_{N}$ nor the phases $\delta_{k}$ contain any measurable PNC terms. Although the $\beta$ state may be coupled to another state by a matrix element $W$ of the weak interaction, the resultant perturbations on the decay rates and energies contained in $a_{N}$ and $\delta_{k}$ are of negligibly small order $|W|^{2}$. Measurable PNC terms, first order in $W$, can occur only in the coupling factors $C_{n}$ in eq. (10).

We note that if the coupling interaction $V_{\beta \alpha}$ in eq. (6) results from an $\mathrm{rf}$ field oscillating at circular frequency $\omega$, then the coupling factor $C_{n}$ shows resonant behavior at $\omega_{\alpha \beta}-\omega=\Omega_{\alpha \beta}=0$. For example, for a single cavity with an rf field which is of uniform intensity along the beam path, we find from evaluation of $C_{\beta \alpha}(\tau)$ of eq. (6),

$$
\begin{aligned}
\left|C_{\beta \alpha}(\tau)\right|^{2}= & \frac{4\left|V_{\beta \alpha}\right|^{2} \mathrm{e}^{-\gamma_{\alpha \beta} \tau}}{\Omega_{\alpha \beta}^{2}+\gamma_{\alpha \beta}^{2}} \\
& \times\left[\sin ^{2} \frac{1}{2} \Omega_{\alpha \beta} \tau+\sinh ^{2} \frac{1}{4} \gamma_{\alpha \beta} \tau\right]
\end{aligned}
$$

Here $V_{\beta \alpha}$ is now time-independent. Eq. (12) shows a reasonance maximum at $\Omega_{\alpha \beta}=0$, which is of size

$\left|C_{\beta \alpha}(\tau)\right|_{\text {MAX }}^{2}=\left|V_{\beta \alpha}\right|^{2}\left(\tau^{2} \mathrm{e}^{\frac{1}{2} \gamma_{\alpha \beta} \tau}\right)[\sinh x / x]^{2}$,

where $x=\gamma_{\alpha \beta} \tau / 4$. Notice that this increases with the "length" $\tau$ of the cavity, but the increase is damped by the decay factor in $\gamma_{\alpha \beta}$.

\subsection{General features of $N$-cavity experiments}

With these remarks we can use the result of (eq. (10) to look at some general features of multiple cavity experiments. First, suppose all $N$ cavities are identical: all coupling factors $C_{n}^{\circ}=\mathrm{C}$ and all phases $\delta_{k}=\delta$. The final $\beta$ state intensity for such an experiment is

$$
\begin{aligned}
\left|\beta_{N}\right|^{2} & \simeq\left\{a_{N}|C|^{2}\right\}\left|\sum_{n=1}^{N} \exp [-\mathrm{i}(n-1) \delta]\right|^{2} \\
& =\left\{a_{N}|C|^{2} \mathrm{e}^{(N-1) \operatorname{Im} \delta}\right\}|\sin N \delta / \sin \delta|^{2} .
\end{aligned}
$$

This produces no more PNC information than is contained already in the single cavity coupling factor $C$, since neither $a_{N}$ nor $\delta$ contains measurable PNC terms. The sine factor in eq. (14), which is identical in form to an optical interference factor, results in an interference effect which narrows the $\alpha \rightarrow \beta$ resonance line by a factor $\sim N$, and increases its intensity by a factor $\sim N^{2}$. The apparent increase in intensiy is damped, however, by the additional decay factor in $\operatorname{Im} \delta$, and the overall intensity change in going from one to $N$ identical cavities is essentially the same as that resulting from simply lengthening a single cavity by a factor $N$ [see eq. (13)]. The line narrowing effect also can be reproduced in essence by lengthening a single cavity. Although both these effects are desirable, they do not represent intrin- sic advantages for multiple cavity PNC experiments over suitably scaled up single cavity schemes. What we have learned about $N$ cavity experiments here is that to provide or new ways of processing PNC information, the cavity couplings $C_{n}$ must differ from one another.

It is not sufficient if the $C_{n}$ differ only by rf field phases. For cavities which are the same except for such phases, one again finds that all the PNC information is contained in the single cavity coupling factor $C$, and the rf phase $\phi_{n}$ only allow us to adjust the size of the signal. We are thus led to consider an $N$ cavity experiment where the couplings $C_{n}$ alternate between production of a "large" PC amplitude and a "small" PNC amplitude. With $\alpha$ and $\beta$ as hydrogen 2S states, a PC amplitude can be generated in one cavity by an Ml (magnetic dipole) transition $\alpha \rightarrow \beta$ driven by an appropriate rf magnetic field. The PNC amplitude can be generated in a second cavity as follows. The state $\beta_{0}\left(F=0, m_{F}=0\right)$ is mixed with the $2 \mathrm{P}$ state $\mathrm{e}_{0}\left(F=1, m_{F}=0\right)$ by the PNC weak interaction. We represent this coupling by: $\mathrm{i} V_{\mathrm{w}}=$ $\left\langle\mathrm{e}_{0}\left|V_{\text {WEAK }}\right| \beta_{0}\right\rangle$, noting that the matrix element is pure imaginary in electroweak theories which preserve time reversal invariance [7]. If, to augment the $\beta_{0}-\mathrm{e}_{0}$ mixing, we add a Stark electric field $E_{\mathrm{s}}$ with pure real matrix element: $V_{\mathrm{s}}=\left\langle\mathrm{e}_{0}\left|e \boldsymbol{E}_{\mathrm{s}} \cdot \boldsymbol{r}\right| \beta_{0}\right\rangle$, then the parity-mixed $\beta$ state is - to first order in $V_{\mathrm{s}}$ and $V_{\mathrm{w}}$

$|\beta\rangle=\left|\beta_{0}\right\rangle+\left|\mathrm{e}_{0}\right\rangle\left[\left(V_{\mathrm{s}}+\mathrm{i} V_{\mathrm{w}}\right) /\left(\omega_{\beta \mathrm{e}}+\frac{1}{2} \mathrm{i} \gamma\right)\right]$,

where $\omega_{\beta c}$ is the $\beta_{0}-\mathrm{e}_{0}$ energy difference and $\gamma$ is the $2 \mathrm{P}$ natural decay rate. With this parity admixture, we can also drive $\alpha \rightarrow \beta$ via an El (electric dipole) transition with an appropriate rf electric field. For example, the $2 \mathrm{P}$ state $\mathrm{e}_{0}$ is coupled to the $2 \mathrm{~S}$ state $\alpha_{0}\left(F=1, m_{F}=0\right)$ by an $\mathrm{rf}$ electric field $\epsilon$ parallel to the Zeeman field (quantization axis), so that there is an $\alpha \rightarrow \beta$ matrix element

$$
\begin{aligned}
& \left\langle\beta|e \epsilon \cdot r| \alpha_{0}\right\rangle=p\left\langle\mathrm{e}_{0}|e \epsilon \cdot \boldsymbol{r}| \alpha_{0}\right\rangle, \\
& P=\left(V_{\mathrm{s}}-\mathrm{i} V_{\mathrm{w}}\right) /\left(\omega_{\beta \mathrm{c}}+\frac{1}{2} \mathrm{i} \gamma\right) .
\end{aligned}
$$

Here, in forming the parity mixing parameter $p$, we have treated the decaying $2 \mathrm{P}$ state by Bell's prescription [15]. A cavity with such electric fields present will generate a PNC amplitude proportional to the small fraction $p$.

\subsection{Prototype two-cavity experiments}

The prototype experiment which follows from this description is diagrammed in fig. 3 . The first cavity has a coupling $C_{\mathrm{M}}$ which generates a "large" $\alpha \rightarrow \beta \mathrm{PC}$ amplitude by an M1 transition, for example, while the second cavity generates a "small" $\alpha \rightarrow \beta$ PNC amplitude by the type of E1 transition just described. To emphasize that the rf field phases $\theta$ and $\phi$ of $C_{\mathrm{M}}$ and $C_{\mathrm{E}}$ may be varied, and that $C_{\mathrm{E}}$ is smaller than $C_{\mathrm{M}}$ by the 


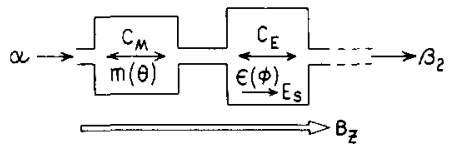

Fig. 3. Prototype PC-PNC experiment. A beam of atoms, initially in the $\alpha$ state, first enters a cavity which generates a "large" $\alpha \rightarrow \beta$ PC amplitude via a coupling $C_{\mathrm{M}}$. This is followed by a second cavity which generates a "small" $\alpha \rightarrow \beta$ PNC amplitude via a coupling $C_{\mathrm{E}}$. The emergent $\beta$ amplitude is $\beta_{2}$, and the alternation of $C_{\mathrm{M}}$ and $C_{\mathrm{E}}$ cavities can be continued. For $\alpha$ and $\beta$ hydrogen $2 \mathrm{~S}$ states, the coupling $C_{M}$ can be provided by an if magnetic field $m(\theta)$ parallel to the local Zeeman magnetic field $B_{z}$, and $C_{E}$ provided by an $\mathrm{rf}$ electric field $\epsilon(\phi)$ also parallel to $B_{z}$. $C_{E}$ can be augmented by a Stark field $E_{\mathrm{s}}$ parallel to $B_{z}$. The phases $\theta$ and $\phi$ of the rf fields can be independently varied.

parity-mixing parameter $p$, we write the couplings as

$C_{\mathrm{M}}=M \mathrm{e}^{-\mathrm{i} \theta}, \quad C_{\mathrm{E}}=p E \mathrm{e}^{-\mathrm{i} \phi}$.

We note that although $M$ and $E$ here are proportional to the respective of field strengths, they also contain the integrals over the field distributions, as in eq. (6), which give the $\alpha \rightarrow \beta$ resonance lineshapes. If we restrict this experiment to two such cavities, the final $\beta$ state intensity is

$$
\left|\beta_{2}\right|^{2} \simeq a_{2}\left|M \mathrm{e}^{-\mathrm{i} \theta}+p E \mathrm{e}^{-\mathrm{i}\left(\phi+\delta_{M}\right)}\right|^{2}=R+J,
$$

where

$$
\begin{aligned}
& R=a_{2}|M|^{2}, \\
& J=2 a_{2} \operatorname{Re}\left\{p M^{*} E \exp \left[-\mathrm{i}\left(\phi-\theta+\delta_{M}\right)\right]\right\} .
\end{aligned}
$$

Here $\delta_{M}$ is the quantum mechanical phase difference characteristic of the $C_{M}$ cavity. An immediate feature of this result is that the relative rf phase $(\phi-\theta)$ can be used as a subtraction variable, since the PNC interference term $J$ changes sign relative to the main signal $R$ when $(\phi-\theta)$ is cycled from 0 to $\pi$. This is a "clean" subtraction since $R$ does not depend on the rf phases (as long as the cavities operate independently) and so the residual $\Delta R$ generated during a phase change is zero in principle.

Several other features of this prototype two cavity experiment are worth mentioning here. First, the sensitivity $S=J / R$ is given by

$S=2 \operatorname{Re}\left\{(p E / M) \exp \left[-\mathrm{i}\left(\phi-\theta+\delta_{M}\right)\right]\right\}$.

This may be optimized not only by varying the relative rf phase $(\phi-\theta)$, but also by suppressing the PC coupling $M$ relative to the PNC coupling $E$-simply by changing the independent cavity parameters which determine $M$ and $E$. Second, the integration time of eq. (3) is

$T=1 / 4 f a_{2}\left[\operatorname{Re}\left\{p E \mathrm{e}^{-\mathrm{i} \alpha_{M}} \exp \left[-\mathrm{i}\left(\phi-\theta+\delta_{\mathrm{M}}\right)\right]\right\}\right]^{2}$,

where $\alpha_{M}=\arg (M)$. The important point here is that $T$ is independent of the magnitude of $M ; T$ is determined mainly by the operating conditions in the $C_{\mathrm{E}}$ cavity, where the PNC amplitude is generated. This means that we can increase the main signal $R=a_{2}|M|^{2}$, generated in the $C_{M}$ cavity, without affecting the integration time. Also, $T$ can be optimized independently by varying the PNC coupling $E$ and the phase $(\phi-\theta)$. Finally, all these features are independent of the details (e.g. specific field geometries) of the coupling factors $C_{\mathrm{M}}$ and $C_{\mathrm{E}}$; it is necessary only that $C_{M}$ in the first cavity produces a $\mathrm{PC}$ amplitude, while $C_{\mathrm{E}}$ in the second cavity produces a PNC amplitude. Altogether, this prototype multiple cavity experiment already demonstrates most of the general features and relative advantages of such experiments as anticipated in the discussion of sect. 2. The appropriate numerical standards $(R \sim 10 \mathrm{ppm}, S \sim$ few ppm, $T \sim 1$ $\mathrm{h} / C^{2}$ ) quoted in sect. 2 also can be achieved for practical designs. What remains is to look ways of improving on this simple prototype.

One shortcoming of the two-cavity experiment just described is that if the PC and PNC coupling factors $C_{M}$ and $C_{E}$ result from just one if field in each cavity, then there are only two subtraction variables available: the rf phase difference $(\phi-\theta)$ and the Zeeman magnetic field $B_{z}$ (note: with reference to fig. 3, reversing $B_{z}$ changes the sign of the pseudoscalar $\boldsymbol{\epsilon} \cdot \boldsymbol{B}_{z}$ which occurs in $C_{E}$; this can also be done by shifting the phase $\phi$ from 0 to $\pi$ ). Since more than two subtraction variables are desirable, we can consider putting more than one field into each of the two cavities described above. Although additional fields can provide new subtraction variables, there are two immediate drawbacks: (1) it is somewhat difficult, technically, to build multi-field cavities, (2) as subtraction variables, the new. fields may suffer from being imperfectly reversible (in both magnitude and direction along the beam path); in the subtraction procedure to isolate the PNC term $J$ from the main signal $R$, this leads to the generation of unacceptably large residuals $\Delta R$ which compete with $J$. Also, in an example to be considered in sect. 4 , the new fields may couple the rf phases into $R$ as well as $J$; this generates residuals $\Delta R$ during phase changes, unlike the example in eq. (19). Altogether, it seems better to create new subtraction variables by adding more easily controllable If phases. To avoid multi-field cavities, this requires going to $N>2$ cavities. 


\subsection{Comparison of two-cavity and $N>2$ cavity experi- ments}

In sect. 4 , we shall consider a specific three-cavity experiment, of the $C_{\mathrm{M}}-C_{\mathrm{E}}-C_{\mathrm{M}}$ type, which has a subtraction variables the Zeeman field $B_{z}$ and two relative rf phases. We shall conclude this section by returning to the question of whether there is any advantage in going to a "large" number of cavities. Previously, in eq. (14), we found that for $N$ identical cavities there was an interesting interference effect, but that the effect could be reproduced in essence by lengthening a single one of the cavities by a factor $N$. Now we shall consider an $N$ cavity experiment where the cavities are not identical, in order to see if the interference effect is modified in an advantageous way.

The experiment we look at is an extension of the prototype in fig. 3: we simply alternate $C_{\mathrm{M}}$ and $C_{\mathrm{E}}$ cavities up to an $N$ th cavity $C_{\mathrm{M}}$, with $N$ an odd number $\geqslant 3$. For simplicity, we assume all the $C_{M}$ cavities have the same coupling $M$, but we allow for different if phases $\theta_{2 n-1}, n=1, \ldots, \frac{1}{2}(N+1)$. Similarly, we let all the $C_{\mathrm{E}}$ cavities have the same coupling $E$, but different rf phases $\phi_{2 n}, n=1, \ldots, \frac{1}{2}(N-1)$. Then the final $\beta$ state intensity is

$$
\left|\beta_{N}\right|^{2} \simeq a_{N}\left|M\left[\Sigma_{\mathrm{M}}\right]+p E \mathrm{e}^{\cdot i \delta_{\mathrm{M}}}\left[\Sigma_{\mathrm{E}}\right]\right|^{2},
$$

where

$$
\begin{aligned}
& \Sigma_{\mathrm{M}}=\sum_{n=1}^{L_{2}^{\prime}(N+1)} \exp \left\{-\mathrm{i}\left[\theta_{2 n-1}+(n-1) \Delta\right]\right\}, \\
& \Sigma_{\mathrm{E}}=\sum_{n=1}^{\frac{1}{2}(N-1)} \exp \left\{-\mathrm{i}\left[\phi_{2 n}+(n-1) \Delta\right]\right\},
\end{aligned}
$$

and $\Delta=\delta_{M}+\delta_{E}$ is the quantum mechanical phase difference accumulated in passing trough a $C_{\mathrm{M}}$ cavity followed by a $C_{E}$ cavity. The sensitivity for this experiment is determined by

$$
\left(A_{\mathrm{PNC}} / A_{\mathrm{PC}}\right)_{N}=(p E / M) \mathrm{e}^{-\mathrm{i} \delta_{\mathrm{M}}}\left[\Sigma_{\mathrm{E}} / \Sigma_{\mathrm{M}}\right] \text {. }
$$

This can be compared with the same ratio for the prototype two cavity experiment of eq. (18), namely,

$$
\left(A_{\mathrm{PNC}} / A_{\mathrm{PC}}\right)_{2}=(p E / M) \mathrm{e}^{-\mathrm{i} \delta_{\mathrm{M}}}\left[\mathrm{e}^{-\mathrm{i}(\phi-\theta)}\right] \text {. }
$$

We see that the $N$ cavity experiment will be significantly different from the 2 cavity experiment only if the factors in square brackets are significantly different.

The rf phase factor in eq. (25) can be changed in sign by cycling $(\phi-\theta)$ from 0 to $\pi$, while for the $N$ cavity experiment this sign change can be accomplished by, for example, all $\theta_{2 n-1}=\theta$, all $\phi_{2 n}=\phi$, and cycling $(\phi-\theta)$. It is also possible to make the magnitude of the ratio $\left[\Sigma_{E} / \Sigma_{M}\right]$ larger than unity. This improves the experimental sensitivity, but only at the expense of choosing an exotic set of rf phases which would be difficult to control. It is far easier to control changes in magnitude in $\left(A_{\mathrm{PNC}} / A_{\mathrm{PC}}\right)$ by varying the power levels in the $C_{\mathrm{M}}$ and $C_{\mathrm{E}}$ cavities. We conclude that the major advantage in going from 2 to $N$ cavities is in adding $(N-2) \mathrm{rf}$ phases as new subtraction variables. For a practical choice of these phases, the experimental sensitivity (and count rate and integration time) for 2 versus $N$ cavities is not likely to change dramatically.

To summarize this section, we have developed a general method for approximately analyzing beam experiments using multiple cavities, and have applied it to the design of hydrogen PNC experiments. We have found that the expected interference effect for $N$ cavities offers no intrinsic advantages over single cavity experiments unless the $N$ cavity couplings differ from one another in both magnitude and phase. Based on this, we have examined a prototype two cavity experiment with variable rf phases which shows most of the features and advantages of such experiments as discussed in sec. 2 . Finally, we have argued that in going beyond two cavities, the only significant advantage lies in introducing new rf phases as subtraction variables.

\section{Examples of multiple cavity PNC experiments}

In this section, we shall describe several specific multiple cavity hydrogen beam PNC experiments in somewhat more detail than was provided by the general discussion of sec. 3 . We do this not only to provide examples of such experiments which may be workable, but also to point out systematic errors which may occur in using rf phases as subtraction variables. The arguments of section 3 lead us to consider only two or three cavity problems. We first discuss general systematic uncertainties which result from the fact that the rf phases always occur in combination with the quantum mechanical phases $\delta_{k}$ of eq. (8), and that the $\delta_{k}$ are uncertain to some degree in a typical beam experiment. Next, we discuss two multi-field experiments with $N=2$ cavities where the rf phases play distinctly different roles as subtraction variables. We then describe an $N=3$ cavity experiment which is an extension of the prototype experiment treated in sect. 3.3.

\subsection{Systematic phase uncertainties}

In the multiple cavity problem, we have introduced the rf field phases as simple exponential phase factors in the cavity couplings $C_{n}$; this is permissible in the rotating wave approximation. Referring to the general final $\beta$ state intensity of eq. (10), we see then that an rf phase $\phi_{n}$ always occurs in combination with the quantum mechanical phases $\delta_{k}$ of eq. (8). The operative overall phase of the $\beta$ state amplitude generated in the $n$th 
cavity is

$\Phi_{n}=\phi_{n}+\sum_{k=1}^{n-1} \delta_{k}$

We shall assume that the rf phases $\phi_{n}$ can be adequately controlled and measured (say to $\Delta \phi_{n} \sim 1^{\circ}$ or $2^{\circ}$ ), and look at effective phase uncertainties associated with the quantum mechanical phases $\delta_{k}$.

We shall ignore the contribution to $\delta_{k}$ from the decay terms, since these terms are relatively insensitive to the effects we shall discuss, and since they ultimately appear in real factors $\exp \left(\operatorname{Im} \delta_{k}\right)$ affecting the size rather than overall phase of the amplitudes. Further, for simplicity, we shall represent the real part of the sum in eq. (26) by a single term: $\operatorname{Re} \delta \simeq \Omega_{\alpha \beta}(L / v)$, where $\Omega_{\alpha \beta}=\omega_{\alpha \beta}-\omega, v$ is the beam velocity, and $L$ is an effective length for the beam transit through a succession of cavities. Then, within a given cavity, there can be effective phase uncertainties $\Delta \Phi$ associated with variations in the following quantity

$\Delta \Phi \sim \Delta(\operatorname{Re} \delta) \simeq \Delta\left[\Omega_{\alpha \beta}(L / v)\right]$.

To fix some numbers, we note that in the hydrogen PNC experiments done near the $\beta_{0}-\mathrm{e}_{0}$ crossing point (Zeeman field $B_{z} \simeq 552 \mathrm{G}$ ), the $\alpha \rightarrow \beta$ frequency separation is $\omega_{\alpha \beta} \sim 2 \pi \times 1550 \mathrm{MHz}$, and the rf cavity operates at frequency $\omega \simeq \omega_{\alpha \beta}$. The $\alpha \rightarrow \beta$ resonance linewidth is $\sim 1 \mathrm{MHz}$, so it is reasonable to assume that under typical operating conditions, the off-resonance frequency in eq. (27) will not exceed $\Omega_{\alpha \beta} \sim 2 \pi \times \frac{1}{2} \mathrm{MHz}$. The metastable beam, produced by $500 \mathrm{eV}$ protons incident on a Cs charge-exchange cell, has a velocity $v \simeq 30$ $\mathrm{cm} / \mu \mathrm{s}$. The effective beam interaction length $L$ may vary from about $10 \mathrm{~cm}$ up to $50 \mathrm{~cm}$ and more; we shall take $L \simeq 30 \mathrm{~cm}$ as typical: Then the quantum mechanical phase in eq. (27) can be as large as: $\operatorname{Re} \delta \sim 180^{\circ}$.

Two simple effects which can cause significant phase uncertainties $\Delta \Phi$ are frequency and mechanical instabilities in the cavities. If the cavity frequency drifts or jitters by only $5 \mathrm{kHz}$, i.e., 3.2. ppm of the $1550 \mathrm{MHz}$ operating frequency, then $\Delta \Phi \simeq 1.8^{\circ}$. If the effective length $L$ changes by $1 \mathrm{~mm}$, then $\Delta \Phi \simeq 0.6^{\circ}$. Fortunately, it should not be difficult to achieve stabilities better than these. Frequency control to better than $1 \mathrm{ppm}$, and mechanical rigidity to better than $1 / 2 \mathrm{~mm}$ reduce the associated phase uncertainties to $\Delta \Phi<1^{\circ}$.

Another phase uncertainty is associated with the beam velocity distribution. The initial proton beam has an energy spread of several percent; this translates to a metastable beam velocity spread $\Delta v / v \sim 1 \%$ at least, and an associated spread in the phase $\Phi$ of $\sim 2^{\circ}$ or so. This sort of phase spread is essentially unavoidable so long as "standard" sources (i.e. duoplasmatron-Cs charge exchange) are used, and it somewhat reduces the sharpness with which phase reversals or phase shifts may be carried out. Also, if the beam velocity drifts or jitters by $\pm 1 \%$, phase uncertainties $\Delta \Phi \sim \pm 2^{\circ}$ may result.

A potentially far more serious source of phase uncertainty comes from the fact that the phase $\Phi$ depends on the Zeeman magnetic field $B_{z}$ through the $\alpha \rightarrow \beta$ frequency separation $\omega_{\alpha \beta}$. For the $\alpha \rightarrow \beta$ transition usually employed (i.e., $\alpha_{0}\left(F=1, m_{F}=0\right) \rightarrow \beta_{0}\left(F=0, m_{F}\right.$ $=0$ ) near $\left.B_{z} \simeq 554 \mathrm{G}\right), \omega_{\alpha \beta}$ has a strong $B_{z}$ dependence: $\partial \omega_{\alpha \beta} / \partial B_{z}=2 \pi \times 2.8 \mathrm{MHz} / \mathrm{G} \simeq 1000^{\circ} \mathrm{MHz} / \mathrm{G}$, so that for beam transit times $L / v \simeq 1 \mu \mathrm{s}$, the phase uncertainty due to an uncertainty $\Delta B_{z}$ in the Zeeman field is - in degrees

$\Delta \Phi=\left(\partial \Phi / \partial B_{z}\right) \Delta B_{z} \simeq 0.56 \times 10^{6}\left(\Delta B_{z} / B_{z}\right)$.

To keep this $\Delta \Phi$ within acceptable bounds requires rather strict homogeneity and control of the Zeeman field. Even for carefully constructed fields which are homogeneous to within $\left(\Delta B_{z} / B_{z}\right) \sim 10-20 \mathrm{ppm}$ along the beam path, there are phase spreads of $\Delta \Phi \sim 5-10^{\circ}$. More importantly, if both $B_{z}$ and $\Phi$ are to be used as subtraction variables, then during a $B_{z}$ reversal good to $\sim 10 \mathrm{ppm}$ (which is difficult to achieve in practice), there will be an inadvertent shift in the operating phase by $\Delta \Phi \sim 5^{\circ}$. This can rule out the use of $B_{z}$ as a subtraction variable in any multiple cavity experiment where the phase $\Phi$ must be controlled within narrower limits.

Altogether, the various sources of phase uncertainties and phase spreads discussed here indicate that it is adequate to control and measure the rf phases $\phi$ to well within the systematic uncertainty $\Delta \Phi \sim 5^{\circ}$. This seems readily possible [12]. On the other hand, our discussion also indicates that an acceptable design for a multiple cavity hydrogen PNC experiment using rf phases as subtraction variables should not be sensitive to uncertainties of just this size. We shall now look at several examples of the use of of phases in $N=2$ and 3 cavity experiments.

\subsection{Multi-field two-cavity experiments}

If we assume that the technical difficulties of building multi-field cavities can be overcome, and that the added fields can be accurately reversed in both magnitude and direction along the beam path, then it is worthwhile to look at what improvements multi-field two cavity experiments may offer over single-cavity schemes.

For our first example, we mention a two-cavity experiment, suggested by Trainor [6], which is a variation of the experiment in progress at the University of Michigan [7,11]. The operative fields in the Michigan single cavity experiment are shown in fig. 4 . A beam of metastable atoms in the state $\alpha=\alpha_{0}\left(F=1, m_{F}=0\right)$ enters the cavity along a Zeeman field $B_{z}$ where it 
encounters a Stark field $E_{x}$ perpendicular to $B_{z}$ and an rf electric field $\epsilon$ inclined at a small angle $\varphi \sim 5^{\circ}$ relative to $B_{z}$. An $\alpha \rightarrow \beta=\beta_{0}\left(F=0, m_{F}=0\right) \mathrm{PC}$ amplitude is

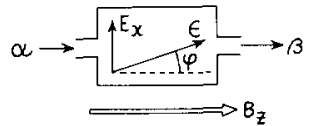

Fig. 4. University of Michigan single cavity experiment. A beam of $\alpha$ state atoms enters along the direction of the Zeeman field $B_{z}$ and encounters a Stark field $E_{x}$ perpendicular to $B_{z}$ and an If electric field $\epsilon$ inclined at an angle $\varphi$ to $B_{z}$. An $\alpha \rightarrow \beta$ PC amplitude is generated by the combination of $E_{x}$ and $\epsilon_{x}=\epsilon \sin$ $\varphi$, while a PNC amplitude results from the weak interaction plus $\epsilon_{z}=\epsilon \cos \varphi$. The subtraction variables for this experiment are $B_{z}, E_{x}$, and $\varphi$, where $\varphi$ is varied by mechanically rotating the cavity.

generated by the combined fields $E_{x}$ and $\epsilon_{x}=\epsilon \sin \varphi$, while a PNC amplitude is generated simultaneously by the weak interaction plus $\epsilon_{z}=\epsilon \cos \varphi$. The subtraction variables for this experiment are $B_{z}, E_{x}$ and $\varphi$, where $\varphi$ is varied by mechanical rotation of the cavity. In the two-cavity version of this experiment, fig. 5 , the fields are essentially the same, except $E_{x}$ and $\epsilon_{x}$ are confined to the first cavity, while $\epsilon_{z}$ is separately applied in the second cavity, where it has an adjustable rf phase $\phi$ relative to $\epsilon_{x}$. A Stark field $E_{z}$ parallel to $B_{z}$ may be added in the second cavity as a probe field for the PNC amplitude generated there. This two cavity experiment is similar to the prototype discussed in sect. 3.3 , in that a "large" $\alpha \rightarrow \beta$ PC amplitude is generated in the first cavity, while a "small" PNC amplitude is separately generated in the second cavity.

Symbolically, the final $\beta$ state intensity for the experiment of fig. 5 can be written as

$$
|\beta|^{2} \simeq a_{2}\left\{\left|k E_{x} \epsilon_{x}\right|^{2}+2 \operatorname{Re}\left[p k^{*} E_{x} \epsilon_{x} \epsilon_{z} \mathrm{e}^{-i(\phi+\delta)}\right]\right\},
$$

where $k$ is a conplex number with dimensions of (electric field) $)^{-1}, p$ is the parity mixing fraction of eq. (16), and $\delta$ is the quantum mechanical phase accumulated in

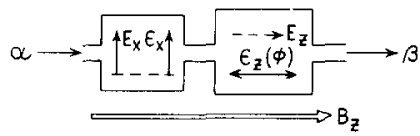

Fig. 5. Two cavity version of Michigan experiment of fig. 4. The fields are the same, except $E_{x}$ and $\epsilon_{x}$ (which together generate the $\alpha \rightarrow \beta$ PC amplitude) are confined to the first cavity, while $\epsilon_{z}(\phi)$ (which together with the weak interaction generates the PNC amplitude) appears separately in the second cavity. A Stark field $E_{z}$ parallel to $B_{z}$ can be added in the second cavity, and the $\mathrm{rf}$ phase $\phi$ of $\epsilon_{z}$ relative to $\epsilon_{x}$ can be varied electronically. The subtraction variables are $B_{z}, E_{x}, \phi$. the first cavity. The subtraction variables for this experiment are $B_{z}, E_{x}$, and the rf phase $\phi$. The potential improvement offered by this two cavity experiment over the single cavity scheme of fig. 4 is the replacement of the mechanically adjusted orientation angle $\varphi$ by an electronically cycled $\mathrm{rf}$ phase $\phi$. We note that the main resonance signal $R$ is independent of $\phi$, so that residuals $\Delta R$ generated during the $\phi$ cycling are zero in principle, and the experiment is relatively insensitive to the phase uncertainties discussed above. With two cavities each of length $\simeq 24 \mathrm{~cm}$, this experiment has a main signal $R \simeq 10 \mathrm{ppm}$ and sensitivity $S \simeq 3 \mathrm{ppm}$ under optimum conditions. By comparison, the Michigan experiment has $R \simeq 5 \mathrm{ppm}, S \simeq 5 \mathrm{ppm}$ for a single cavity $\simeq 11 \mathrm{~cm}$ long.

For our second example, we describe an experiment of a type now under construction at the University of Washington [16]. This two cavity scheme is portrayed in fig. 6. As in most of the hydrogen PNC experiments, the transition studied is between the $2 \mathrm{~S}$ hfs levels $\alpha_{0}(F=1$, $\left.m_{F}=0\right)$ and $\beta_{0}\left(F=0, m_{F}=0\right)$. The transition is driven in each cavity by a combination of a Stark field $E_{k}$ and rf electric field $\epsilon_{k}$; this generates both a PC and PNC amplitude in each cavity. The Stark fields can be varied in both magnitude and direction. The rf fields can be varied in magnitude, and the relative $\mathrm{rf}$ phase $\phi$ between $\epsilon_{1}$ and $\epsilon_{2}$ can be cycled electronically. The principal subtraction variables for this experiment are $B_{2}, E_{1}$ (for $\left.E_{2} \ll E_{1}\right)$, and the rf phase $\phi$.

The coupling factors in each cavity contain both a Stark matrix element $V$ and weak interaction matrix element $\mathrm{i} V_{\mathrm{w}}$. The final $\beta$ state intensity may be written as

$|\beta|^{2} \simeq\left\{a_{2} \mathscr{L}\left(\omega_{\beta \mathrm{c}}\right) \mathrm{e}^{\operatorname{Im} \delta}\right\}|F(\Phi)-\mathrm{i} G(\Phi)|^{2}$,

where

$F(\Phi)=V_{1} \epsilon_{1} \mathrm{e}^{\mathrm{i} \Phi / 2}+V_{2} \epsilon_{2} \mathrm{e}^{-\mathrm{i} \Phi / 2}$,
$G(\Phi)=V_{\mathrm{w}}\left(\epsilon_{1} \mathrm{e}^{\mathrm{i} \Phi / 2}+\epsilon_{2} \mathrm{e}^{-\mathrm{i} \Phi / 2}\right)$.

Here $a_{2}$ is the attenuation factor for the two cavities,

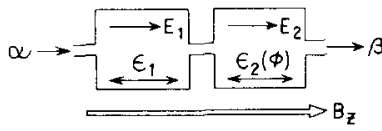

Fig. 6. University of Washington double cavity experiment. A beam of $\alpha$ state atoms enters along the Zeeman field $B_{z}$ and traverses two similar cavities where $\alpha \rightarrow \beta$ transitions are driven by a combination of Stark fields $E_{k}$ and rf electric fields $\epsilon_{k}$, all parallel to $B_{z}$. Each cavity generates both a PC and PNC amplitude. The Stark fields can be varied in both magnitude and direction. The rf fields can be varied in magnitude, and the relative phase $\phi$ between $\epsilon_{1}$ and $\epsilon_{2}$ can be electronically cycled. The principal subtraction variables for the experiment are $B_{z}$. $E_{1}$, and $\phi$. 
$\mathcal{L}\left(\omega_{\beta \mathrm{e}}\right)=1 /\left(\omega_{\beta \mathrm{e}}^{2}+\frac{1}{4} \gamma^{2}\right)$ is a Lorentzian function which is resonant at the $\beta_{0}-\mathrm{e}_{0}$ crossing point, $V_{1}$ and $V_{2}$ are the Stark matrix elements associated with the dc electric fields $E_{k}, \epsilon_{1}$ and $\epsilon_{2}$ are proportional to the rf field strengths and also contain integrals over the cavity $\mathrm{rf}$ couplings, and $\Phi=\phi+\delta$ is the effective phase between the two cavities, with $\delta$ the quantum mechanical phase accumulated in the first cavity. $F(\Phi)$ is proportional to the net PC amplitude generated in the two cavities, while $G(\Phi)$ is proportional to the net PNC amplitude. The main signal $R$ and PNC interference term $J$ for this experiment are

$$
\begin{aligned}
& R(\Phi) \simeq\left\{a_{2} \mathcal{L}\left(\omega_{\beta \mathrm{e}}\right) \mathrm{e}^{\operatorname{Im} \delta}\right\}|F(\Phi)|^{2}, \\
& J(\Phi) \simeq 2\left\{a_{2} \mathcal{L}\left(\omega_{\beta \mathrm{e}}\right) \mathrm{e}^{\operatorname{Im} \delta}\right\} \operatorname{Re}\left[\mathrm{i} F^{*}(\Phi) G(\Phi)\right] .
\end{aligned}
$$

We note immediately that as long as all four electric fields (i.e., the Stark fields $E_{1}$ and $E_{2}$, and rf fields $\epsilon_{1}$ and $\epsilon_{2}$ ) are non-zero, then both $R$ and $J$ depend upon the phase $\Phi=\phi+\delta$.

The dependence of the main signal $R$ on the phase $\Phi$ can lead to problems with using the rf phase $\phi$ as a subtraction variable, because of the phase uncertainties discussed in sect. 4.1 above. We can demonstrate this by setting: $V_{1} \epsilon_{1}=-V_{2} \epsilon_{2}, \epsilon_{1}=\epsilon_{2}=\epsilon$. Then

$$
\begin{aligned}
R(\Phi) \simeq & 4 V_{1}^{2}|\epsilon|^{2}\left\{a_{2} \mathcal{L}\left(\omega_{\beta \mathrm{e}}\right) \mathrm{e}^{\operatorname{Im} \delta}\right\}|\sin (\Phi / 2)|^{2}, \\
J(\Phi) \simeq & 8 V_{\mathrm{w}} V_{1}|\epsilon|^{2}\left\{a_{2} \mathcal{E}\left(\omega_{B \mathrm{e}}\right) \mathrm{e}^{\operatorname{Im} \delta}\right\} \\
& \times \operatorname{Re}\left[\sin \left(\Phi^{*} / 2\right) \cos (\Phi / 2)\right] .
\end{aligned}
$$

The sensitivity is given by

$S=J / R=2\left(V_{\mathrm{w}} / V_{1}\right) \operatorname{Re}[\operatorname{ctn}(\Phi / 2)]$.

Suppressing $R$ to give an adequate $S \simeq$ few ppm requires either applying relatively small Stark fields or choosing an operating point with the phase $\operatorname{Re} \Phi \rightarrow 0$. If a Stark field $E \sim 1 \mathrm{~V} / \mathrm{cm}$ is taken as a sort of natural lower limit ( $E$ should be "large" compared to systematic stray and motional electric fields which may be present), then: $S \simeq 0.2 \operatorname{Re}[\operatorname{ctn}(\Phi / 2)] \mathrm{ppm}$. Clearly, further suppression of $R$ is necessary by adjusting $\Phi ; \operatorname{Re} \Phi$ should be held at less than $10^{\circ}$ or so. But such an accurate fix on $\Phi$ is difficult to obtain in view of the phase uncertainties $\Delta \Phi \sim 5^{\circ}$ discussed in sect. 4.1. Also, an operating point $\operatorname{Re} \Phi \rightarrow 0$ near the minimum in $R(\Phi)$ generates potentially large main signal residuals during any subtraction procedure which introduces some uncertainty in $\Phi$ (e.g., either the $\phi$ or $B_{z}$ subtraction); if the uncertainty is $\Delta \Phi$, the fractional main signal residual is

$\Delta R / R \simeq|\operatorname{ctn}(\Phi / 2)| \Delta \Phi$.

This is of order unity for $\operatorname{Re} \Phi \sim 10^{\circ}$ and $\Delta \Phi \sim 5^{\circ}$. Under these conditions, the use of both the if phase $\phi$ and Zeeman field $B_{z}$ as subtraction variables is virtually ruled out. Altogether, this particular version of the present two-cavity experiment is too sensitive to phase uncertainties to be practicable. From the standpoint of using rf phases as subtraction variables, we see that: (1) if possible, the same rf phase should not appear in both the main signal $R$ and PNC interference term $J,(2)$ and rf phase should not be used to suppress $R$ at the same time it is being used as a subtraction variable.

These difficulties can be circumvented in the experiment of fig. 6 by letting the Stark field $E_{2} \rightarrow 0$ in the second cavity. According to eqs. (31) and (32), this weakens the $\Phi$ dependence of $R$ (but does not remove it entirely unless $E_{2} \equiv 0$ ), while preserving the $\Phi$ dependence of $J$. The sensitivity is thereby reduced ( $S \propto \operatorname{Re}[\sin$ $\Phi]$ rather than $S \propto \operatorname{Re}[\operatorname{ctn}(\Phi / 2)])$, but this loss may be recovered by adjusting the rf electric fields so that $\left|\epsilon_{2}\right|>\left|\epsilon_{1}\right|$. This is what is planned for the Washington experiment [16], where a main signal $R \simeq 3 \mathrm{ppm}$ and sensitivity $S \simeq 7 \mathrm{ppm}$ are calculated for two cavities which are $12 \mathrm{~cm}$ and $36 \mathrm{~cm}$ long respectively.

Other examples of multi-field two cavity hydrogen PNC experiments can be given. For example, Hinds has suggested a two cavity scheme for studying transitions $\alpha \rightarrow \beta$ with $\Delta m_{F}=1$ rather than the usual $\Delta m_{F}=0$ [6]. The analysis of such experiments is similar to what we have done here, however, and so we shall not go into further detail. Generally, these experiments have three subtraction variables: the Zeeman magnetic field $B_{z}$, some Stark field $E$, and a relative phase $\phi$ between rf electric fields in the separate cavities. They have main signals $R$ and sensitivities $S$ which are comparable to those of the best single cavity experiments. In fact, all these experiments have roughly the same value of the product $R S^{2}$, so that by eq. (3) they all have about the same integration time. The two cavity experiments typically have total interaction lengths $L \sim 50 \mathrm{~cm}$, so that some effort is necessary to build suitably homogeneous Zeeman fields over this length $\left(\Delta B_{z} / B_{z} \sim 20 \mathrm{ppm}\right.$ is nominal). As well, the cavities must be carefully lined up with each other and with the Zeeman field (alignment to within $10^{-3} \mathrm{rad}$ is nominal). If these specifications were to be met, the two cavity experiments offer one advantage over single cavity schemes by the introduction of the rf phase $\phi$ as a subtraction variable. $\phi$ can be rapidly and accurately cycled, and even though the effective phase $\Phi=\phi+\delta$ is subject to systematic uncertainties (principally through its dependence on $B_{z}$ ), it should be possible to carry out $\phi$ subtractions which separate the PNC interference term from the main signal without generating main residuals in principle. Thus, $\phi$ can be a sort of "super" subtraction variable, as compared with others which generate residuals unless they are perfectly reversible.

In passing, we note that the major source of phase uncertainty in these types of experiments is the $B_{z}$ dependence of $\Phi$, as calculated in eq. (28) for hydrogen $\alpha \rightarrow \beta$ transitions. This may be largely eliminated by choosing a transition whose Bohr frequency separation 
depends only weakly on $B_{z}$ (i.e $\partial \omega_{\alpha \beta} / \partial B_{z} \rightarrow 0$ ). Thus, Hinds has suggested studying transitions between hfs levels in the hydrogen $\beta$ state [17]. This has the advantage of removing the rather strict requirements on magnetic field homogeneity and control

\subsection{A two-phase three-cavity experiment}

We have already mentioned, in sect. 3.4 , that each time we add a new cavity we obtain another if phase which is potentially usable as a subtraction variable. If we are satisfied with three subtraction variables altogether, and start from a two-cavity experiment with the set $\left(B_{z}, \phi, E\right)$ just described, we can ask what advantage might be gained by going to a three-cavity scheme with the set $\left(B_{z}, \phi, \theta\right)$, where the new $\mathrm{rf}$ phase $\theta$ replaces the Stark field $E$ as a subtraction variable. Of course, we wish to keep the rf phase $\phi$ as a "super" subtraction variable, as in the two-cavity experiments. The requirements on $\theta$, as a replacement for $E$, are less stringent; $\theta$ need not be a "super" subtraction variable. This is because $E$ is not "super"; $E$ normally enters both the main signal $R$ and the PNC interference term $J$, so that residuals may be generated during an imperfect $E$ reversal. The relative advantages of replacing a Stark field $E$ by an rf phase $\theta$ then may be judged on the basis of the following comparisons: (1) relative size of residuals $\Delta R$ for $\theta$ vs $E$ cycling, (2) control and uniformity of $\theta$ vs. $E$ cycling, (3) relative ease in building a $\left(B_{z}, \phi, E\right)$ vs $\left(B_{z}\right.$, $\phi, \theta)$ experiment. For purposes of comparison, we shall now discuss a three-cavity experiment of the latter type which has been described previously $[6,18]$.

Before going into detail, we make some qualitative judgements regarding the above comparisons. First, construction problems do not appear to particularly favor one method over the other. Although building and lining up a third cavity present difficulties, they are probably no worse than building and lining up suitable dc electrodes within a cavity. The overall interaction lengths $(L \sim 50 \mathrm{~cm})$ can be made approximately the same for both the two- and three-cavity schemes, so the design of the Zeeman field region is not much different. Second, as $\theta$ is a scalar while $E$ is a vector, the latter intrinsically requires more control than $\theta$ under a given reversal. Third, regarding point (1) above, we note that in the two cavity experiments described by eqs. (29) and (33), $E$ appears in the main signal $R$ in such a way that its value must be set within rather narrow limits in order to adequately suppress $R$. As we have seen, it is usually not desirable to use a subtraction variable also as a supression variable. In the three-cavity experiment we are about to describe, this problem does not occur. Finally, because of Stark quenching of final state metastables, eliminating $E$ in favor of an rf phase $\theta$ may allow larger final-state signal strengths.

The triple-cavity scheme which follows from these considerations is a natural extension of the prototype two-cavity experiment already described in sec. 3.3, eqs. (17)-(21), and fig. 3. We show the cavity configuration in fig. 7. The first and third cavities are essentially identical, and they generate $\alpha \rightarrow \beta$ PC amplitudes via $\Delta m_{F}=0 \mathrm{Ml}$ transitions induced by rf magnetic fields $m_{1}(0)$ and $m_{2}(\theta)$ parallel to the Zeeman field $B_{z}$. The first cavity has a reference of phase of zero, and the relative phase $\theta$ of the third cavity is variable. An $\alpha \rightarrow \beta$ PNC amplitude is generated in the central cavity by a combination of the weak interaction and an rf electric field $\epsilon(\phi)$ again parallel to $B_{z}$. The relative if phase $\phi$ of $\epsilon(\phi)$ is independently variable, and a "probe" Stark field $E_{s} \| B_{z}$ may be added in the central cavity if desired; $E_{\mathrm{s}}$ is not necessary. The subtraction variables for this experiment are the set $\left(B_{z}, \phi, \theta\right)$.

The cavity couplings for this experiment can be represent by $C_{\mathrm{M}}$ and $C_{\mathrm{E}}$ as in eq. (17). The emergent $\beta$ state intensity is

$|\beta|^{2} \simeq a_{3}\left|M_{1}+p E \mathrm{e}^{-\mathrm{i} \Phi}+M_{2} \mathrm{e}^{i(\Theta)}\right|^{2}$,

where

$\Phi=\phi+\delta_{M}, \delta_{M} \simeq\left[\Omega_{\alpha \beta} \tau\right]_{M} ;$
$\Theta=\theta+\left(\delta_{M}+\delta_{E}\right)$,
$\delta_{E} \simeq\left[\left(\Omega_{\alpha \beta}-\frac{1}{2} \mathrm{i} \gamma_{\alpha \beta}\right) \tau\right]_{\mathrm{E}}$.

We write out the quantum mechanical phases $\delta_{\mathrm{M}}$ and $\delta_{\mathrm{E}}$ for the $C_{\mathrm{M}}$ and $C_{\mathrm{E}}$ cavities (assuming negligible drift time between them) to emphasize the fact that there is negligible quenching of the metastables in the Ml cavities, so that $\delta_{\mathrm{M}}$ is (very nearly) pure real. The only significant quenching is by $\alpha \rightarrow \mathrm{e}$ transitions in the central cavity, and the attenuation factor $a_{3}=$ $\exp \left[-\left(\gamma_{\alpha \beta} \tau\right)_{\mathrm{E}}\right]$. The main signal $R$ and PNC interference term $J$ are

$$
\begin{aligned}
& R(\Theta)=a_{3}\left|M_{1}+M_{2} \mathrm{e}^{-\mathrm{i} \Theta}\right|^{2}, \\
& J(\Theta, \Phi)=2 a_{3} \operatorname{Re}\left[p\left(M_{1}+M_{2} \mathrm{e}^{-\mathrm{i} \Theta}\right) * E \mathrm{e}^{-\mathrm{i} \Phi}\right] .
\end{aligned}
$$

We see that the central cavity phase $\phi$ is a "super"

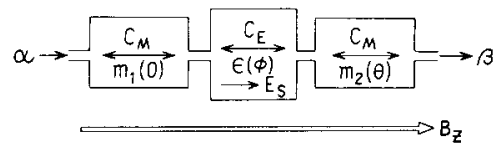

Fig. 7. Triple cavity M1-El experiment. This is an extension of the prototype two cavity experiment in fig. 3 . The first and third cavities generate $\alpha \rightarrow \beta$ PC amplitudes via rf magnetic fields $m_{1}(0)$ and $m_{2}(\theta)$ which are parallel to the Zeeman field $B_{z}$. The relative phase $\theta$ between $m_{1}$ and $m_{2}$ is variable. The central cavity generates an $\alpha \rightarrow \beta$ PNC amplitude via an rf electric field $\epsilon(\phi)$, also parallel to $B_{z}$, whose phase $\phi$ relative to $m_{1}$ is independently variable. A "probe" Stark field $E_{s}$ may be added (but is not necessary) in the central cavity. The subtraction variables for this experiment are $B_{z}, \phi$, and $\theta$. 
subtraction variable since $R$ is independent of $\phi$. The Zeeman field $B_{z}$ is also an admissible (non "super") subtraction variable, since the PNC term $J$ contains the pseudoscalar $E \propto \boldsymbol{\epsilon} \cdot \boldsymbol{B}_{z}$.

As for the effect of varying the second if phase $\theta$, this can be seen more clearly by assuming the M1 cavities are run at the same power level, so that $M_{2}=$ $M_{1}=M$, and

$$
\begin{gathered}
R(\Theta)=4|M|^{2}\left\{a_{3} \mathrm{e}^{\operatorname{Im} \delta_{\mathrm{E}}}\right\}|\cos (\Theta / 2)|^{2}, \\
J(\Theta, \Phi)=4 a_{3} \operatorname{Re}\left\{p M^{*} E \cos \left(\Theta^{*} / 2\right)\right. \\
\left.\times \exp \left[-\mathrm{i}\left(\Phi-\frac{1}{2} \Theta^{*}\right)\right]\right\} .
\end{gathered}
$$

Assuming for the moment that $\Theta$ is real, $R(\Theta)$ is an even function of $\Theta$, while $J$ contains terms which are odd in $\Theta$. A subtraction can then be carried out under the phase reversal $\Theta \rightarrow(-) \Theta$. The fact that $\Theta$ is complex does not change the substance of this remark and the phase $\Theta$ can be used as a subtraction variable (with appropriate offsets to cancel $\operatorname{Re}\left(\delta_{M}+\delta_{E}\right)$ etc. $)$. Of more importance are the residuals $\Delta R$ generated by $\Theta$ phase uncertainties during the setting or reversal of the rf phase $\theta$.

We note that the integration time $T$ is independent of the Ml cavity power level $\propto|M|^{2}$, so that $R \propto|M|^{2}$ can be run at any desired rate. Also, adequate sensitivity can be provided by adjusting $|M|$, so that the phase $\Theta$ need not be used as a suppression variable. This means that we can run at the maximum of $R$ vs. $\Theta$. The fractional residual due to an uncertainty $\Delta \Theta$ is then

$\Delta R / R=|\tan (\Theta / 2)| \Delta \Theta$.

For even "large" phase uncertainties $\Delta \Theta \sim 5^{\circ}$ around $\operatorname{Re} \Theta \sim 0$, we have $\Delta R / R \sim \frac{1}{2}(\Delta \Theta)^{2}<1 / 250$, an acceptable residual when other subtraction variables are available. It is much smaller than the comparable quantity in eq. (35) because here we are allowed to operate at the maximum rather than minimum of the $R$ vs. $\Theta$ curve. The triple-cavity experiment should be then relatively insensitive to the seemingly large phase uncertainties of sect. 4.1.

The new rf phase $\Theta$ is thus an acceptable subtraction variable. We can compare $\Theta$ quantitatively with the Stark field $E$ it is meant to replace by noting that in those multi-field two cavity experiments where the main signal $R \propto E^{2}$, the fractional residual generated during an $E$ reversal with uncertainty $\Delta E$ is: $\Delta R / R=2 \Delta E / E$. To achieve the nominal value of $\Delta R / R<1 / 250$, which seems readily possible for $\theta$ reversals, we need to control an $E$ reversal down to $\Delta E<E / 500$, or $2 \mathrm{mV} / \mathrm{cm}$ out of a $1 \mathrm{~V} / \mathrm{cm}$ field. Although this is being done [11], it may be somewhat easier to achieve the same level of discrimination by phase reversals. For this reason, and the qualitative reasons mentioned earlier, the two-phase triple-cavity experiment would seem to have advantages over multi-field double cavity schemes.

We remark finally that the triple-cavity experiment can achieve "standard" values for main signal strength $R(\simeq 10 \mathrm{ppm})$, sensitivity $S(\simeq 3 \mathrm{ppm})$, and integration time $T\left(\simeq 1 \mathrm{~h} / C^{2}\right)$ for practical designs (two $\mathrm{Ml}$ cavities each $19 \mathrm{~cm}$ long, one El cavity $20 \mathrm{~cm}$ long). This experiment also has the feature that since $R$ is not limited by Stark-induced quenching, it may be increased (essentially arbitrarily) to improve the signal/background ratio if necessary. Although this does decrease the sensitivity of the experiment, it does not affect the integration time. With an optimum value of the rf electric field in the central El cavity, $S \propto \tau_{\mathrm{E}}$ and $T \propto$ $1 / \tau_{E}$, so the experiment may be improved by increasing the transit time $\tau_{\mathrm{E}}$ for this cavity.

\section{Summary and conclusions}

In this paper, we have attempted to develop some general guidelines and criteria for designing and evaluating multiple cavity hydrogen beam PNC experiments, starting from the existing single cavity designs. By an approximate analysis which allows comparison of various experimental schemes on an essentially equal footing, we have found that for multiple cavities the expected Ramsey interference effect provides new methods of processing PNC data. The new methods involve shifting the relative phases of the rf fields between separated cavities which contain distinctly different field configurations that are designed to separately generate PC (parity conserving) and PNC (parity nonconserving) transition amplitudes. Since these rf phases can be rapidly and accurately cycled, there are advantages in using them to replace PNC data processing "subtraction" variables which are more difficult to control and/or cycle. Our general analysis also indicates that other than gaining rf phases as subtraction variables, there appears to be no intrinsic advantage in going to an experiment with $N=$ "large" number of cavities; $N=2$ or 3 suffices for hydrogen PNC experiments. Finally, we have enumerated various sources of systematic errors in controlling and setting phases, and have looked at how phases may be used in several specific $N=2$ and 3 cavity designs.

Although the final choice of an experimental design may be made on the basis of minimizing systematic effects which we have not considered here, we can draw the following conclusions regarding multiple- versus single-cavity PNC experiments and the use of rf phases as subtraction variables:

1) Appropriately designed multiple-cavity PNC experiments do not give significantly better (or worse) sensitivities and integration times than existing singlecavity schemes; in some cases, however, the main signal 
rate $R$ may be increased to improve the signal/background ratio.

2) Multiple-cavity schemes do allow simpler designs for individual cavities (e.g. cavities need not be rotatable, and need not contain dc electrodes); the overall interaction region length is increased, however, which requires design of longer homogeneous Zeeman field regions.

3) As subtraction variables, the rf phases in multiple-cavity PNC experiments seem to offer some advantage over those available in single-cavity schemes, even though the phases are subject to inevitable systematic uncertainties; for example, there are "super" subtraction phases which can be used to process the PNC data without generating any competing main signal residual $\Delta R$ in principle.

4) Multiple-cavity PNC experiments can be designed which are relatively insensitive to phase uncertainties (but still allow suitably accurate phase subtractions), and which do not require using the rf phases to suppress the main signal $R$; in such experiments, using rf phases to replace as many field subtraction variables as is practicable may be preferable.

For these reasons, multiple-cavity hydrogen beam PNC experiments have considerable appeal.

One of us (R.T.R.) wishes to thank E.T.H. Zürich for the hospitality and support extended to him during his visit there, and to express his gratitude to the University of Michigan for providing a continuing place of refuge. Both authors have benefited from discussions with many friends and/or colleagues; among them are: E.A. Hinds, L.P. Lévy, R.R. Lewis and V.L. Telegdi. This work was supported in part by the National Science Foundation, under grants PHY79-25044 and ISP80-11449.

\section{References}

[1] L. Wolfenstein, Proc. Int. Workshop on Neutral current interactions in atoms, Cargèse (1979) p. 32.
[2] M.A. Bouchiat and C.C. Bouchiat, J. Phys. (Paris) 35 (1974) 899

[3] P. Bucksbaum, E. Commins and L. Hunter, Phys. Rev. Lett. 46 (1981) 640 .

J.H. Hollister et al., Phys. Rev. Lett. 46 (1981) 643 and references therein

[4] W.L. Williams, 5th Int. Symp. on Polarization phenomena in nuclear physics, Santa Fe (August 1980) p. 1384.

[5] N.F. Ramsey, Molecular beams (Oxford Press, 1956) sect V.4.

[6] E.A. Hinds, Proc. 6th Int. Workshop on Weak interactions with very high energy beams, Ames (1978) p. 151; T.A Trainor, Cargèse Proc., ref. 1, p. 231; E.A. Hinds, ref. 1, p. 256; R.T. Robiscoe, ref. 1, p. 275.

[7] R.W. Dunford, R.R. Lewis and W.L. Williams, Phys. Rev. Al 8 (1978) 2421.

[8] R.R. Lewis and W.L. Williams, Phys. Lett. B59 (1975) 70

[9] E.A. Hinds and V.W. Hughes, Phys. Lett. B67 (1977) 487

[10] E.G. Adelberger, T.A. Trainor and E.N. Fortson, Bull. Am. Phys. Soc. 23 (1978) 546

[11] We note, however, that electric field reversal with $\Delta R \leqslant$ $10^{-3}$ has been achieved, L.P. Lévy and W.L. Williams, Phys. Rev. Lett. 48 (1982) 607.

[12] Appropriate phase shifters $\left(\Delta \phi=360^{\circ}\right.$ at $1-2 \mathrm{GHz}$, with rapid cycling) are available commercially. See, e.g., Cata$\log$ '79 from Triangle Microwave, 11 Great Meadow Lane, East Hanover, NJ 07936, U.S.A.

[13] E.A. Hinds, Phys. Rev. Lett. 44 (1980) 374.

[14] W.E. Lamb, Jr., and R.C. Retherford, Phys. Rev. 79 (1950) 549; W.E. Lamb, Jr., Phys. Rev. 85 (1952) 259.

[15] J.S. Bell, Cargèse Proc., ref. 1, p. 288. The role of the $2 \mathrm{P}$ state damping in hydrogen parity experiments was first discussed by Lewis and Williams, ref. 8. An experimental test of the role of damping in single cavity schemes is being carried out, see L.P. Lévy and W.L. Williams, Phys. Rev. Lett. 48 (1982) in press.

[16] E.G. Adelberger, et al., Nucl. Instr. and Meth. 179 (1981) 181.

[17] E.A. Hinds, private communication.

[18] R.T. Robiscoe, ETH Zürich memorandum, Status Report on the ETHZ Hydrogen PNC Experiment (July 1979) unpublished. 